\title{
Dynamic Data Communications for Real-Time Information Fusion
}

\author{
Weichang Wang ${ }^{1}$ and Lei Ying ${ }^{2}$ \\ ${ }^{1,2}$ Arizona State University, Tempe, AZ, 85287, USA \\ wwang195@asu.edu \\ lei.ying.2@asu.edu
}

\begin{abstract}
This paper studies dynamic data communications between airplanes and a control tower, where the control tower needs to monitor the state of each aircraft close to the airport or on the ground in real time. Given limited communication bandwidth, it is impossible for the control tower to communicate with all aircrafts at the same time. This paper focuses on the problem of optimal scheduling of data communications for the control tower to acquire information from aircrafts to minimize tracking errors. A dynamic learning problem with limited communication bandwidth is formulated in this paper where the objective is to minimize the total variance of real-time tracking. To solve the problem, a dynamic scheduling algorithm for data communications is proposed, which prioritizes data communications based on the tracking variances of the aircrafts, channel conditions and importance of the information. Our simulations demonstrate that our algorithm outperforms policies such as a round robin policy.
\end{abstract}

\section{INTRODUCTION}

Communications play a vital role in safety and operations of air transportation systems. While voice communications is the main means in current air transportation systems, digital communications are essential to facility real-time information exchange and improve situation awareness. In general, a control tower can only communicate with a subset of aircrafts nearly due to limited bandwidth. Therefore, it is imperative to have an efficient resource allocation mechanism to schedule data communications to minimize tacking errors with limited communication bandwidth.

This paper considers the problem of dynamic data communications for real-time information infusion, which expects to be critical in the next-generation air transportation systems. In particular, we focus on data communications between a control tower and the aircrafts in its controlled space. The

\footnotetext{
Weichang Wang et al. This is an open-access article distributed under the terms of the Creative Commons Attribution 3.0 United States License, which permits unrestricted use, distribution, and reproduction in any medium, provided the original author and source are credited.
}

control tower acquires information from aircrafts to monitor the trajectories of all aircrafts in its controlled space in real time. The goal of the dynamic data communications is to minimize the tracking errors by optimizing the information transmitted to the control tower.

\subsection{Related Work}

The use of Kalman filter to track aircrafts is motivated by (Hwang, Balakrishnan, \& Tomlin, 2006), where the problem of tracking a single aircraft was. Our problem is also related to information management in wireless sensor networks. For example, assume the number of sensors to be activated is fixed in advance, the problem of optimal sensor selection for maximizing the accuracy of a single estimation has been studied in (Joshi \& Boyd, 2009), where a heuristic selection method has been proposed and a bound on the best performance based on convex optimization. This paper focuses on continuous estimation of the states of dynamical systems. (Williams, Fisher, \& Willsky, 2007) studied the tradeoff between communication energy cost and the value of information contained, and proposed an approximate dynamic programming approach for sensor network management. The problem was extended to a more complicated periodic sensor scheduling on a linear time invariant system in (Liu, Fardad, Masazade, \& Varshney, 2014).

\subsection{Notation}

In this paper, the boldface little letters in this paper represent vectors and i.e. $\mathbf{x}(t)$, and capital letters represent matrix or constant i.e. $Q(t)$.

\section{A General Problem formulation}

In this section, we introduce a general problem formulation for real-time information fusion under communication constraints. The model includes a continuous time dynamical system and a discrete time estimator. In particular, we consider the following time-invariant (TIV) dynamical system for each aircraft indexed by $k$ :

$$
\dot{\mathbf{x}}_{k}(t)=\mathbf{f}\left(\mathbf{x}_{k}(t)\right)+\mathbf{w}_{k}(t),
$$


where $\mathbf{x}_{k} \in \mathbb{R}^{N}$ is the state vector of the system at, $\mathbf{f}$ is the system equation and, and $\mathbf{w}_{k}(t)$ is zero mean white-noise process with spectral density matrix $\mathbf{Q}_{k}$. This TIV system is used to model a physical system such as an aircraft, so we chose to use a continuous-time model.

We further assume the control tower keeps track the states of all nearby aircrafts. We assume a discrete-time estimator that updates its estimates every time slot. We chose a discretetime model because most wireless communications systems are time-slotted systems. Therefore, the control tower receives the updates in a discrete-time fashion. Assuming the duration of each time slot is $\tau$ units of time. At time slot $m$, the control tower receives measurement $\mathbf{z}_{k}$ from aircraft $k$ such that

$$
\mathbf{z}_{k}[m]=h_{k}\left(\mathbf{x}(m \tau)+\mathbf{v}_{k}[m]\right),
$$

where $h_{k} \in\{1,0\}$ such that $h_{k}=1$ if aircraft $k$ sends an update to the control tower and $h_{k}=0$ otherwise. In the equation above, $\mathbf{v}_{k}$ is a zero mean Gaussian random variables with associated covariance matrices $R_{k}$, and it can be understood as the measurement error of the sensors.

To model limited wireless bandwidth, we assume the control tower can communicate with $C(C<N)$ aircrafts at the same time. In other words, the following constraint has to hold

$$
\sum_{k=1}^{N} h_{k} \leq C .
$$

To solve the problem, we assume that:

$$
E\left[\mathbf{w}_{l}(t) \cdot \mathbf{v}_{k}[m]\right]=0 \quad \text { for all } k, l, t \text { and } m,
$$

which means that the random forcing noise and measurement noise are independent.

To keep track of the state of an aircraft, the control tower estimates based on the measurements received up to the current time $t$ and calculates

$$
\hat{\mathbf{x}}(t)=E\left[\mathbf{x}(t) \mid \mathbf{z}_{0}, \mathbf{z}_{1}, \cdots, \mathbf{z}_{m}\right] \quad m \tau \leq t
$$

where $\hat{\mathbf{x}}$ is an estimator of $\mathbf{x}(t)$.

Because the state of the system is Gaussian under the assumption of this paper, we consider the following error covariance matrix:

$$
\mathbf{P}(t)=E\left[(\mathbf{x}(t)-\hat{\mathbf{x}}(t))^{T}(\mathbf{x}(t)-\hat{\mathbf{x}}(t))\right]
$$

$\mathrm{t}$ and use $\operatorname{tr}(\mathbf{P}(t))$ as the performance metric of state estimation.

Furthermore, we assume the control tower uses a standard Continuous-Discrete Linearized Kalman filter to monitor all aircrafts. The linearized Kalman filter works as follows:
1. Initialization: The first estimation can be a random value chosen as follows:

$$
\mathbf{x}_{k}(0) \sim \mathcal{N}\left(\hat{\mathbf{x}}_{0}, \mathbf{P}_{0}\right) .
$$

2. Propagation

(a) State Estimation Propagation

$$
\dot{\hat{\mathbf{x}}}_{k}(t)=\mathbf{f}\left(\hat{\mathbf{x}}_{k}(t)\right)
$$

(b) Error Covariance Propagation

$$
\dot{\mathbf{P}}_{k}(t)=\mathbf{F}\left(\hat{\mathbf{x}}_{k}(t)\right) \mathbf{P}_{k}(t)+\mathbf{P}_{k}(t) \mathbf{F}\left(\hat{\mathbf{x}}_{k}(t)\right)+\mathbf{Q}_{k}
$$

where

$$
\mathbf{F}\left(\hat{\mathbf{x}}_{k}(t)\right)=\left.\frac{\partial \mathbf{f}\left(\mathbf{x}_{k}\right)}{\partial \mathbf{x}_{k}}\right|_{\mathbf{x}_{k}=\hat{\mathbf{x}}_{k}(t)}
$$

3. Estimation Update

The gain matrix is defined as follows. If $h_{k}=1$, then

$$
\mathbf{K}_{k}[m]=\mathbf{P}_{k}\left(m \tau^{-}\right)\left(\mathbf{P}_{k}\left(m \tau^{-}\right)+\mathbf{R}_{k}\right)^{-1},
$$

where $\mathbf{P}_{k}\left(m \tau^{-}\right)$is the value of $\mathbf{P}_{k}$ before the state update is received.

(a) State Estimation Update

$$
\hat{\mathbf{x}}_{k}[m]=\hat{\mathbf{x}}_{k}\left(m \tau^{-}\right)+\mathbf{K}_{k}[m]\left(\mathbf{z}_{k}[m]-\hat{\mathbf{x}}\left(m \tau^{-}\right)\right)
$$

where $\hat{\mathbf{x}}_{k}\left(m \tau^{-}\right)$is the estimated state vector at time $m \tau$ before the measurement received, and $\mathbf{x}_{k}[m]$ is the estimated after receiving the update.

(b) Error Covariance Update

$$
\mathbf{P}_{k}[m]=\mathbf{P}_{k}(m \tau)=\left(\mathbf{I}-\mathbf{K}_{k}\right) \mathbf{P}_{k}\left(m \tau^{-}\right) .
$$

It is well-known that $\hat{\mathbf{x}}(t)$ is the optimal estimation of $\mathbf{x}(t)$ in the mean square error sense when the system function $\mathbf{f}(\hat{\mathbf{x}}(t))$ is linear. Assuming the Kalman filter is used, we next study the problem of minimizing the total variance of tracking by designing the scheduling policy for data transmissions, i.e.,

subject to:

$$
\begin{gathered}
\min _{h[1], \cdots, h[m], \cdots} \lim _{N \rightarrow \infty} \frac{1}{N} \sum_{m=1}^{N} \operatorname{tr}\left(\mathbf{P}_{k}[m]\right) \\
\sum_{k=1}^{N} h_{k}[m] \leq C \quad \forall m .
\end{gathered}
$$

\section{Aircraft Tracking Model}

In this section, we first introduce the aircraft tracking model.

Let $N$ denote the total number of aircrafts, $N_{a}$ the number of aircrafts in the air, and $N_{g}$ the number of aircrafts on the ground. The aircrafts in the air and on the group follow different dynamics and the communication channels have different qualities.

Wireless telecommunications often suffer from multipath interference, which results in signals reaching the receiving 


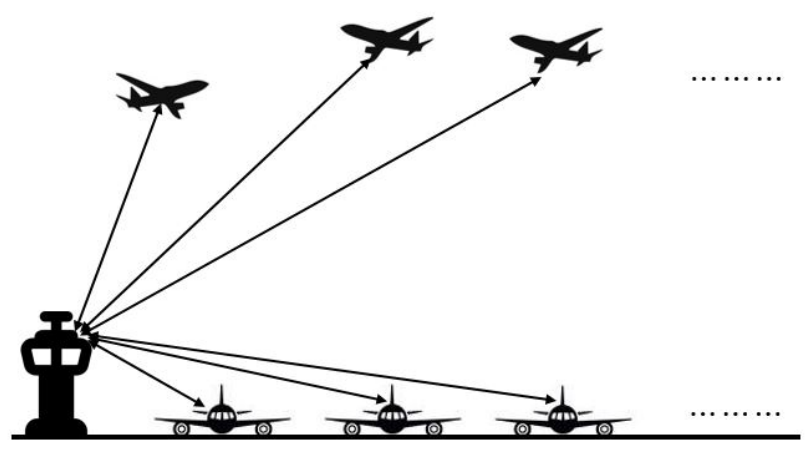

Figure 1. Airport model

antenna from two or more paths. The ratio between the power in the direct path and the power in the other paths is called the Rice factor. According to (Haas, 2002), the Rice factor of en-route scenario and arrival $\backslash$ takeoff scenario is higher that of the taxi scenario and parking scenario, the reason is that the Line of Sight (LOS) component which plays an important role in the multipath interference is often blocked by the buildings while the aircrafts are on the ground. The Rice factor significantly determines the bit error rate of a channel. We assume that the success rate of dat transmissions for aircrafts in the air is $p_{a}=0.99$, and is $p_{g}=0.9$ for the aircrafts on the ground.

\subsection{Taking Off $\backslash$ Landing Aircraft Model}

According to the Kinematic equations for aircraft motions proposed by (P. K. Menon \& Cheng, 2018), as an aircraft taking off, we have following motion equations:

$$
\begin{aligned}
& \dot{h}=f(h, A) \\
& \gamma=\sin ^{-1}\left\{\frac{f(h, A)}{v_{a}}\right\} \\
& \dot{\lambda}=\frac{1}{\left(R_{e}+h\right)}\left(V_{a} \cos \gamma \cos \chi+W_{N}\right) \\
& \dot{\tau}=\frac{1}{\left(R_{e}+h\right) \cos \lambda}\left(V_{a} \cos \gamma \sin \chi+W_{E}\right) \\
& \chi=\tan ^{-1}\left\{\frac{\sin \left(\tau_{n x t}-\tau\right) \cos \lambda_{n x t}}{\sin \lambda_{n x t} \cos \lambda-\sin \lambda \cos \lambda_{n x t} \cos \left(\tau_{n x t}-\tau\right)}\right\}
\end{aligned}
$$

where $(\lambda, \tau)$ is the latitude and longitude of the the aircraft, and $\left(\lambda_{n x t}, \tau_{n x t}\right)$ is the position of the next waypoint in the aircraft flight plan. $\gamma$ is the flight path angle with respect to local horizontal, and $\chi$ is the course angle with respect to true North. $h$ is aircraft altitude relative to mean sea level, $V_{a}$ is airspeed, and $R_{e}$ is the radius of Earth. $f(h, A)$ is the aircraft type-dependent climb rate data in the Base of Aircraft Data (BADA) (Gallo, Navarro, Nuic, \& Iagaru, 2006). $W_{E}$ and $W_{N}$ are the wind speed along east and north, respectively
The state of an aircraft in the air can be modeled as $[h, \lambda, \tau]^{T}$, and we have the following dynamics:

$$
\left[\begin{array}{c}
\dot{h} \\
\dot{\lambda} \\
\dot{\tau}
\end{array}\right]=\left[\begin{array}{c}
f(h, A) \\
\frac{1}{\left(R_{e}+h\right)}\left(V_{a} \sqrt{1-\frac{f(h, A)}{V_{a}}} \cos \chi+W_{N}\right) \\
\frac{1}{\left(R_{e}+h\right) \cos \lambda}\left(V_{a} \sqrt{1-\frac{f(h, A)}{V_{a}}} \sin \chi+W_{E}\right)
\end{array}\right]+\mathbf{w}_{a}
$$

where $\mathbf{w}_{a}$ is the random forcing noise of $[h, \lambda, \tau]^{T}$, with spectral density matrix $Q_{a}(t)$. The landing model can be obtained following similar analysis.

\subsection{Runway Aircraft Model}

Similar to the model discussed in (Khadilkar \& Balakrishnan, 2011), we have the following model for an aircraft on the ground:

$$
\left[\begin{array}{c}
\dot{x} \\
\dot{y} \\
\dot{V}_{g} \\
\dot{\theta}
\end{array}\right]=\left[\begin{array}{c}
V_{g} \sin \theta \\
V_{g} \cos \theta \\
f_{V}(t) \\
f_{\theta}(t)
\end{array}\right]+\mathbf{w}_{g}
$$

where $(x, y)$ is the position of the aircraft on the ground, $V_{g}$ is the velocity, and $\theta$ is the direction the aircraft is heading. $f_{V}(t)$ and $f_{\theta}(t)$ are determined by the movement plan decided by the controller. The forcing noise

$$
\mathbf{w}_{g} \sim \mathcal{N}\left(0,\left[\begin{array}{cccc}
0 & 0 & 0 & 0 \\
0 & 0 & 0 & 0 \\
0 & 0 & \sigma_{w V}^{2} & 0 \\
0 & 0 & 0 & \sigma_{w \theta}^{2}
\end{array}\right]\right)
$$

can be viewed as the operational deviation of the pilot.

Putting the models for the dynamics of aircrafts together, we have

$$
\left\{\begin{array}{l}
\dot{\mathbf{x}}_{a, i}=\mathbf{F}_{a}\left(\mathbf{x}_{a, i}\right)+\mathbf{w}_{a, i} \quad i=1,2, \cdots, N_{a} \\
\dot{\mathbf{x}}_{g, j}=\mathbf{F}_{g}\left(\mathbf{x}_{g, j}\right)+\mathbf{w}_{g, j} \quad j=1,2, \cdots, N_{g}
\end{array}\right.
$$

where $\mathbf{F}_{a}(\mathbf{x})$ and $\mathbf{F}_{g}(\mathbf{x})$ are the system functions defined in (11) and (12), respectively. And the random force noise $\mathbf{w}_{a, i}$ and $\mathbf{w}_{g, i}$ have spectral density matrix $\mathbf{Q}_{a}$ and $\mathbf{Q}_{g}(t)$.

\section{An Adaptive Algorithm for Data Communi- CATIONS}

In this section, we propose an adaptive algorithm to solve this problem defined the previous section. Our numerical evaluation in the next section will show that our algorithm outperforms policies such as Round Robin.

Assume that at time slot $m$, after acquiring information from the aircraft, the updated error covariance of the $i$ th aircraft in the air is $\mathbf{P}_{(a, i)}[m]$, and the covariance of the $j$ th aircraft on the ground is $P_{(g, j)}[m]$. Then at time slot $m+1$, the predicted values of $\mathbf{P}_{(a, i)}\left[(m+1)^{-}\right]$and $\mathbf{P}_{(g, j)}\left[(m+1)^{-}\right]$ 
can be derived according to equation (6). If the $i$ th aircraft in the air is chosen to transmit an update, the expected updated covariance is

$$
\begin{aligned}
E\left[\mathbf{P}_{(a, i)}[m+1] \mid h_{i}=1\right]= & \left(1-p_{a}\right) \mathbf{P}_{(a, i)}\left[(m+1)^{-}\right]+ \\
& p_{a} \mathbf{P}_{(a, i)}\left[(m+1)^{+}\right] .
\end{aligned}
$$

where $\mathbf{P}_{(a, i)}\left[(m+1)^{+}\right]$is the updated error covariance computed from equation (10), and it is the error covariance matrix if the measurement is transmitted to the controller successfully. If the $i$ th aircraft in the air is not chosen, we have:

$$
E\left[\mathbf{P}_{(a, i)}[m+1] \mid h_{j}=0\right]=\mathbf{P}_{(a, i)}\left[(m+1)^{-}\right] .
$$

Similarly, $E\left[\mathbf{P}_{(g, j)}[m+1]\right]$ can be computed condition on whether aircraft $j$ on the group is selected for data transmissions. Based on these, the greedy algorithm can be described as:

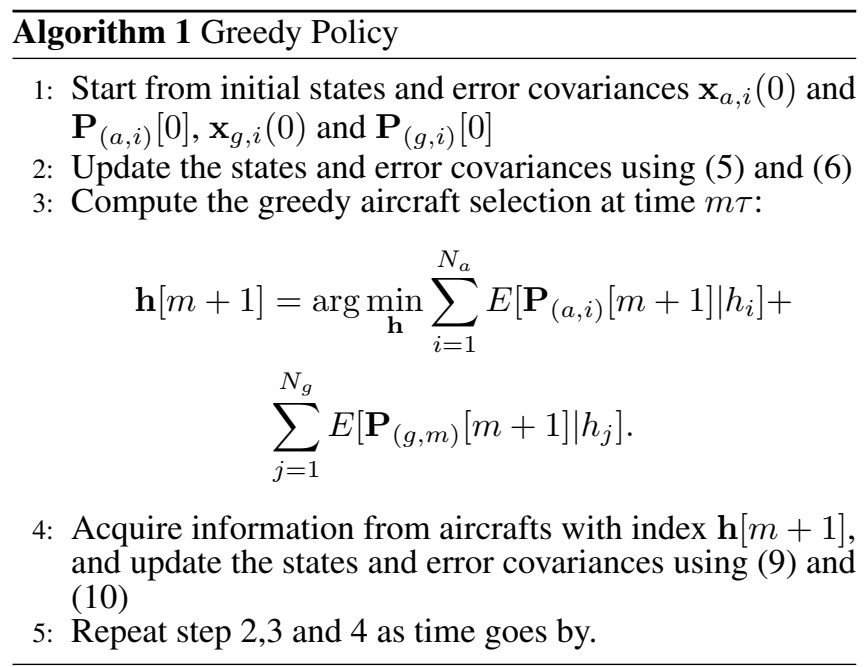

With this greedy algorithm, the communication resources are assigned to the most aircrafts whose information can be used to reduce the total variance.

\section{Simulations}

In this section, we use simulations to demonstrate the proposed adaptive algorithm, and compare it with the roundrobin algorithm. Assume that the aircraft taking off from Phoenix Sky Harbor airport $\left(33.4373^{\circ} N, 112.0078^{\circ} W\right)$, and the next waypoint is at $\left(33.5^{\circ} \mathrm{N}, 112^{\circ} \mathrm{W}\right)$, and they are also initial state of coming aircraft, such that the trajectories of aircrafts in the air can be derived from these two waypoints using (11). For simplicity, we assume that the climb or decrease rate function $f(h, A)$ is a linear function, and that the wind speed is a constant. For the aircraft on the ground, they start from position $(0,0)$, and we assume that their acceleration and the angular acceleration follow some sinusoidal function over time. However, different states might have different importance, for example, in the air model, 0.1 degrees in latitude or longitude can be much more significant than 0.1 meters in height, so we will use weighted sum of variance and error to make the decision. Also according to (Gelb, Staff, \& Corporation, 1974), the optimality of the Kalman filter is independent with the weights. The weighted sum of variance and error are shown in Fig. 2 and Fig3. The average values of the variance and error are shown in Table.1

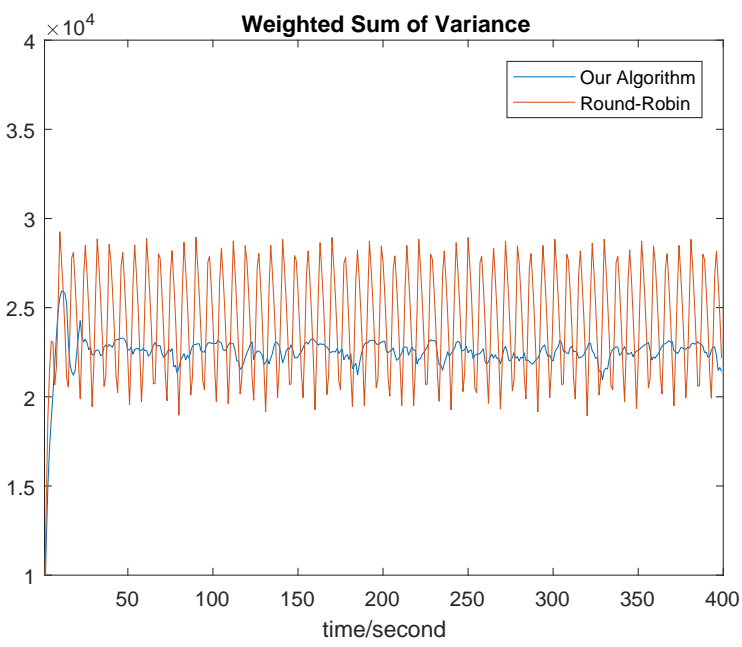

Figure 2. Weighted Sum of Variance

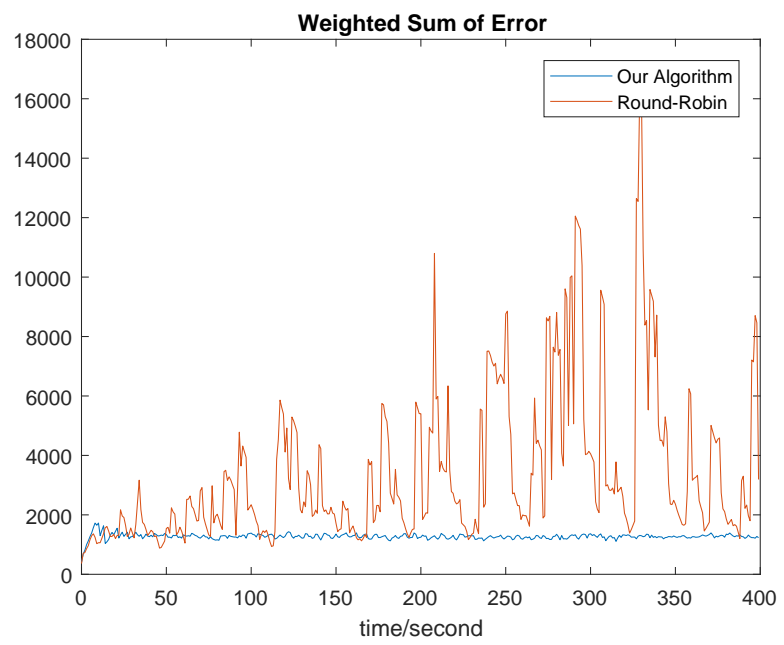

Figure 3. Weighted Sum of Variance

Table 1. Average

\begin{tabular}{|c|c|c|}
\hline & Average Variance & Average Error \\
\hline Greedy Algorithm & $2.2422 \mathrm{e}+04$ & $1.2731 \mathrm{e}+03$ \\
\hline Round Robin & $2.4194 \mathrm{e}+04$ & $3.3853 \mathrm{e}+03$ \\
\hline
\end{tabular}


Also, we show the trajectories of one aircraft in the air and one aircraft on the ground in Fig. 4 and 5 under the roundrobin and our algorithm.

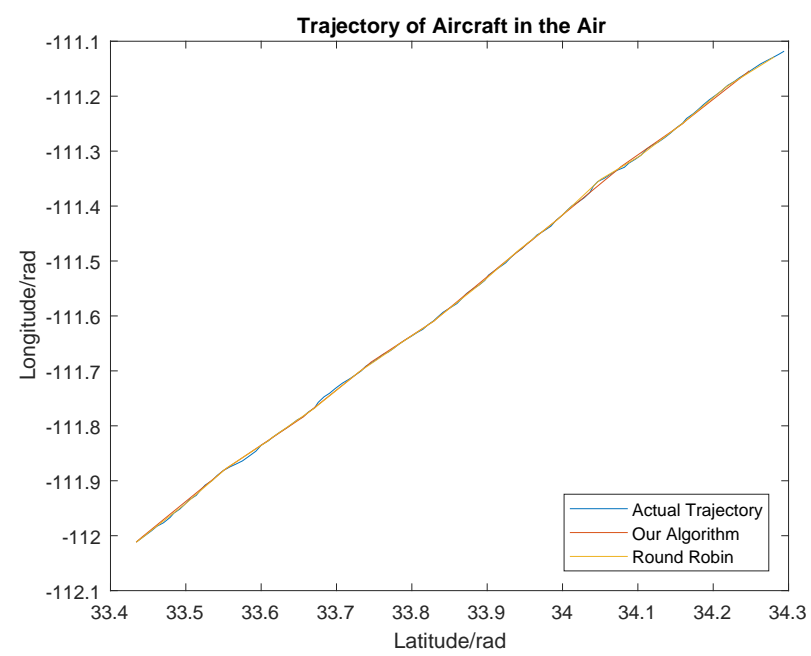

Figure 4. Trajectory of one Aircraft in the Air

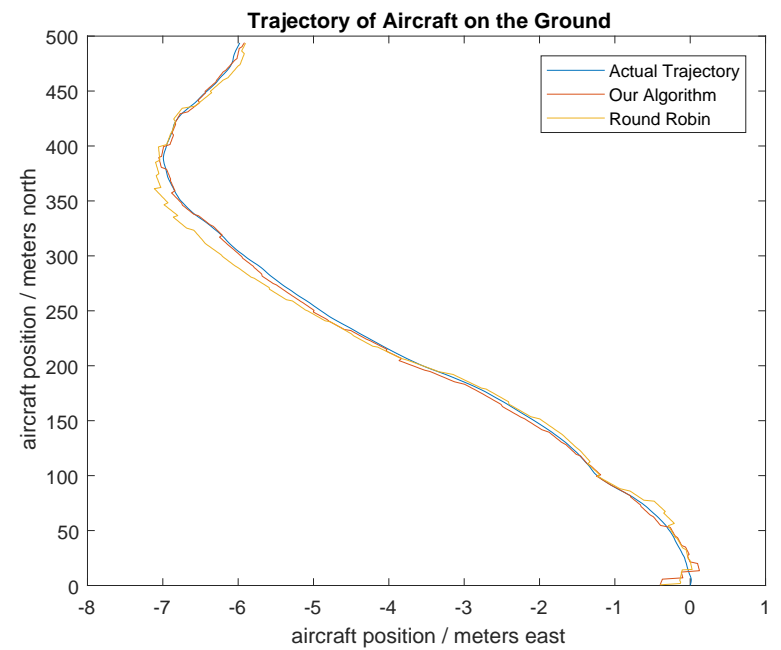

Figure 5. Trajectory of one Aircraft on the Ground

From the numerical results above, we can observe that our algorithm significantly outperform the round robin policy.

\section{Conclusion}

In this paper, we proposed a dynamic data communications algorithm for real-time information fusion. The numerical results demonstrated that outperforms round-robin in both accuracy and variance. The trajectories of two aircrafts showed that the control towel can track the aircrafts more accurately than round-robin communication mechanisms. In our future work, we will incorporate important environmental and geological factors, such as weather, humidity, surface conductivity and so on into the model and the algorithm.

\section{ACKNOWLEDGEMENT}

The research reported in this paper was supported in part by funds from NASA University Leadership Initiative program (Contract No. NNX17AJ86A, Project Officer: Dr. Kai Goebel, Principal Investigator: Dr. Yongming Liu). The support is gratefully acknowledged.

\section{REFERENCES}

Gallo, E., Navarro, F. A., Nuic, A., \& Iagaru, M. (2006). Advanced aircraft performance modeling for atm: Bada 4.0 results. In 2006 ieeelaiaa 25th digital avionics systems conference (p. 1-12). doi: 10.1109/ DASC. 2006.313660

Gelb, A., Staff, A. S. C. T., \& Corporation, A. S. (1974). Applied optimal estimation. MIT Press.

Haas, E. (2002). Aeronautical channel modeling. IEEE Transactions on Vehicular Technology, 51(2), 254-264. doi: $10.1109 / 25.994803$

Hwang, I., Balakrishnan, H., \& Tomlin, C. (2006). State estimation for hybrid systems: applications to aircraft tracking. IEE Proceedings - Control Theory and Applications, 153(5), 556-566. doi: 10.1049/ip-cta: 20050053

Joshi, S., \& Boyd, S. (2009). Sensor selection via convex optimization. IEEE Transactions on Signal Processing, 57(2), 451-462. doi: 10.1109/TSP.2008.2007095

Khadilkar, H., \& Balakrishnan, H. (2011). A multimodal unscented kalman filter for inference of aircraft position and taxi mode from surface surveillance data. 11th AIAA Aviation Technology, Integration, and Operations (ATIO) Conference. doi: 10.2514/6.2011 $-7051$

Liu, S., Fardad, M., Masazade, E., \& Varshney, P. K. (2014). Optimal periodic sensor scheduling in networks of dynamical systems. IEEE Transactions on Signal Processing, 62(12), 3055-3068. doi: 10.1109/TSP .2014 .2320455

P. K. Menon, P. D. S. G. P. O. C., B-J. Yang, \& Cheng, V. H. L. (2018). A computational platform for analyzing the safety of the national airspace system.

Williams, J. L., Fisher, J. W., \& Willsky, A. S. (2007). Approximate dynamic programming for communicationconstrained sensor network management. IEEE Transactions on Signal Processing, 55(8), 4300-4311. doi: 10.1109/TSP.2007.896099 